\title{
Spread of multiresistant strains of Salmonella typhimurium phage types 204 and 193 in Britain
}

Since June 1977 two phage types of Salmonella typhimurium, both showing multiple drug resistance, have caused many infections in bovines and humans throughout Britain. The phage types are type 204, with resistance to chloramphenicol, streptomycin, sulphonamides, and tetracyclines (R-type CSSuT), and type 193, with resistance to ampicillin, chloramphenicol, neomycin-kanamycin, streptomycin, sulphonamides, and tetracyclines (R-type ACKSSuT).

The CSSuT-resistant type 204 strain first appeared in calves in June 1977 and has subsequently caused infections in cattle in many areas of Britain. ${ }^{1}$ Symptoms were of severe scouring and there was a high mortality rate among infected animals. There have been 56 human isolations of type 204 of R-type CSSuT, mostly from cases of mild enteritis. Some cases were in families in farming communities, but many had no obvious connection with farms. The strain has also been isolated from minced beef prepared for human consumption.

In December 1977 strains of $S$ typhimurium phage type 193 of R-type ACKSSuT affected calves on farms in Herefordshire and Worcestershire. Subsequently, infections occurred in bovines in many areas of England, Wales, and Scotland, and the transport of infected animals facilitated this spread. Human infections first occurred in December 1977, and by the end of June 1978 there had been 44 isolations of multiresistant type 193 strains. As with type 204, some infections occurred in farming families, but most had no direct connection :with bovines. So far, there have been no isolations from milk or human foods.

Characterisation of the resistance plasmids in the type 193 strains from both bovine and human sources showed that these strains had an identical plasmid content and represented a single clone. This had been derived from the epidemic phage type 204 strain of R-type CSSuT by the acquisition of an $R$ factor of compatibility group $I_{1}$, coding for resistance to ampicillin, kanamycin, and streptomycin (AKS). This plasmid inhibits the lysis of strains of $S$ typhimurium by several typing phages. When the AKS group $I_{1}$ plasmid is displaced from the multiresistant strains of phage type 193, the resultant lines belong to phage type 204 of $S$ typhimurium, and have the CSSuT R-type and plasmid content of the epidemic strain.

Earlier studies showed that the CSSuT-resistant type 204 strain was derived from a strain of phage type 204 of R-type SuT, by the acquisition of an $R$ factor of compatibility group $\mathrm{H}_{2}$, which codes for chloramphenicol, streptomycin, sulphonamide, and tetracycline resistance (CSSuT). ${ }^{1}$ Type 204 of R-type SuT became the predominant strain in bovine and human $S$ typhimurium infection in 1976, and remained so until the establishment of the chloramphenicol-resistant epidemic type 204 strain, in late 1977 . The possible progenitor of type 204 of R-type SuT was a sulphonamide-resistant strain of phage type 49 , which had acquired a non-autotransferring plasmid of an unidentifiable compatibility group coding for tetracycline resistance. This tetracycline plasmid changed the phage type of type 49 to type 204. ${ }^{1}$ The overall sequence of events is illustrated in the figure.

The progressive acquisition of drug resistance plasmids by a sulphonamide-resistant type 49 strain may have been produced by the

1) Type 49 R-type: $\mathrm{Su}$

T plasmid

Type 204 (compatibility group: unidentifiable)

2) Type 204

CSSuT plasmid

\begin{tabular}{|c|c|}
\hline \multirow{2}{*}{$\begin{array}{l}\text { Type } 204 \\
\text { R-type: } \\
\text { SuT }\end{array}$} & CSSuT plasmid \\
\hline & (compatibility group: $\mathbf{H}_{2}$ ) \\
\hline \multirow{2}{*}{$\begin{array}{l}\text { Type } 204 \\
\text { R-type: } \\
\text { CSSuT }\end{array}$} & AKS plasmid \\
\hline & \\
\hline
\end{tabular}

Plasmid acquisition and phage type change. use of antibacterial drugs in bovine salmonellosis. Firstly, tetracyclines may have acted to produce type 204 of R-type SuT. Subsequently, the use of chloramphenicol, which has been administered for bovine salmonellosis, may have exerted selective pressure to maintain the epidemic CSSuT-resistant type 204 strain in calf herds. Streptomycin and sulphonamides may also have exerted selective pressure, but this is unlikely, since their use in veterinary practice is more limited. Finally, the use of ampicillin or kanamycin in bovines may have contributed to the appearance of the multiresistant phage type 193 strain. Some bovine isolations of the type 193 strain have recently acquired plasmid-encoded resistance to trimethoprim $(\mathrm{Tm})$, which suggests that drugs such as co-trimoxazole are now being used in cattle.

Since 1970 multiresistant salmonellae with enhanced virulence and a tendency to produce septicaemia have caused epidemics in many countries. A multiresistant strain of $S$ wien has spread through paediatric units in countries of Southern Europe and the Middle East, ${ }^{2}$ with a high mortality rate among infected infants. Similarly, a multiresistant strain of $S$ typhimurium phage type 208 has caused many severe infections throughout the Middle East. ${ }^{4}$

The emergence and establishment of the multiresistant type 204 and 193 strains in Britain have been facilitated by the use of antibacterial drugs in attempts to control bovine salmonellosis. More prudence in the use of antibiotics in veterinary practice is necessary to prevent the emergence of other, possibly more virulent strains of resistant salmonellae, which may present a serious threat to human health. Should the multiresistant $S$ typhimurium strains described in this paper cause extra-intestinal infections in humans; the clinician's choice of antimicrobial treatment would be extremely limited.

1 Threlfall, E J, Ward, L R, and Rowe, B, Veterinary Record, 1978, in press. 2 Le Minor, S, Médicine et Maladies Infectieuses, 1972, 2, 441.

${ }_{3}^{3}$ McConnell, M M, Smith, H R, Leonardopoulos, J, and Anderson, E S, Fournal of Infectious Diseases, 1978, in press.

4 Anderson, E S, et al, fournal of Hygiene, 1977, 79, 425.

(Accepted 31 fuly 1978)

Division of Enteric Pathogens, Central Public Health Laboratory, London NW9 5HT

E J THRELFALL, BSC, PHD, senior scientist

L R WARD, BSC, principal scientist B ROWE, MA, MB, director

\section{Thyroid crisis and tracheal compression in patient with retrosternal goitre}

Thyroid crisis is now fortunately rare and usually seen as a complication of a stressful illness. ${ }^{1}$ In cases of true retrosternal goitre, which represent only $1 \%$ of all goitres, ${ }^{2}$ toxicity is uncommon and when present is normally mild. We describe here a patient with retrosternal goitre who had severe toxicity. The condition was difficult to manage because of inadequate initial treatment.

\section{Case report}

A 74-year-old woman was admitted complaining of palpitations, giddiness, and weight loss despite a good appetite. On examination the thyroid gland was enlarged and partially retrosternal. She had a fine tremor and pronounced lid lag. She was in mild congestive cardiac failure with a pulse rate maintained at 80 beats/min by beta-blockade.

Triiodothyronine (T3) concentration was $7.3 \mathrm{nmol} / 1(4.8 \mathrm{ng} / \mathrm{ml}$ ) (normal $1.2-3.0 \mathrm{nmol} / 1(0.78-2.0 \mathrm{ng} / \mathrm{ml}))$; thyroxine (T4) $>290 \mathrm{nmol} / 1(225 \mathrm{ng} / \mathrm{ml})$ (normal 70-90 $\mathrm{nmol} / 1$ (54-70 $\mathrm{ng} / \mathrm{ml}$ )). Antibodies to thyroid microsomal antigen were present in high titre. Technetium scan showed an enlarged 